\title{
Evidence-Based Guideline for Adult Sedation, Pain Assessment, and Analgesia in a Low Resource Setting Intensive Care Unit: Review Article
}

This article was published in the following Dove Press journal: International Journal of General Medicine

\author{
Derartu Neme' \\ Zemedu Aweke (D) \\ Haileleul Micho ${ }^{2}$ \\ Simeneh Mola (D) \\ Bedru Jemal (iD) \\ Teshome Regasa' \\ 'Department of Anesthesiology, College \\ of Medicine and Health Science, Dilla \\ University, Dilla, Ethiopia; ${ }^{2}$ Department \\ of Biochemistry, College of Medicine and \\ Health Science, Dilla University, Dilla, \\ Ethiopia
}

Background: Agitation and anxiety occur frequently in ICU and affect about $30-80 \%$ of patients in ICU present with delirium worldwide, and it is associated with adverse clinical outcomes. This review aimed to systematically review articles and finally draw an evidencebased guideline for an area with limited resources.

Methods: The review was reported based on preferred reporting items for systemic and meta-analysis (PRISMA) protocol. We searched literature from PubMed, Google Scholar, and Medline database using keywords like the level of sedation, sedation score, pain assessment in ICU, and sedative drugs in ICU from an article published in English. After extraction with a patient population and exclusion, five randomized clinical trials, four systemic reviews and meta-analysis, four observation cohort study, and two practical guidelines were used for the review.

Conclusion: In addition to high validity and reliability, RASS has the advantage of easiness to remember for nurses making it a preferred sedation assessment tool in an adult ICU setting. Light sedation with daily interruption was recommended with an aim of an awake and alert patient ready for the weaning trial. Propofol was preferred when sedation is for a short duration and when intermittent awakening is required. Ketamine is the preferred induction for asthmatic hypotensive and patient requiring prolonged continuous sedation. With a similar time for sedation, diazepam shows a shorter time for intubation compared to midazolam. Besides diazepam has shown a cheaper cost of sedation than midazolam. This makes it a drug of preference in a low resource setting.

Keywords: sedation, ICU, propofol, ketamine

\section{Introduction}

Sedation is the act of calming patients by the administration of sedative medications. Sedative and analgesic drugs are commonly administered for ICU patients worldwide because the experience of pain and anxiety occurs in the majority of critically ill patients during ICU stay time. Up to $50 \%$ of surgical and medical ICU patients experience pain at rest and $80 \%$ during procedures or nursing care. Tracheal suctioning, removal of drains, positioning of the patients are reported as among the most painful procedures in ICU. ${ }^{1,2}$

Agitation and anxiety occurs frequently in ICU and also about $30 \%$ to $80 \%$ of patients in ICU present with delirium world-wide and it is associated with adverse clinical outcomes such as difficulty in tracheal suctioning and IV cannulation, increase oxygen consumption, and also it may result in accidental extubation for
Department of Anesthesiology, College of Medicine and Health Science,

Dilla University, P.O. 0419, 4620, Dilla,

Ethiopia

Tel +25l 912406236

Email zemeduawoke@gmail.com
International Journal of General Medicine 2020:13 |445-1452

1445

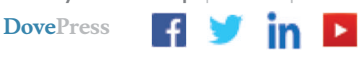

htt://doi.0rg/10.2147/1,GM.S276878 
mechanically ventilated patients. ${ }^{3}$ Early detection and intervention of the possible underlying causes for agitation and anxiety such as pain, delirium, hypoxemia, hypoglycemia, hypotension, or withdrawal from alcohol and other drugs are very important before sedating the patient. ${ }^{4}$

In ICU patients, monitoring of sedation and analgesia has many challenges since they are critically ill patients most of them even may not communicate and cannot report their pain verbally or with other signs due to a diminished level of consciousness, being on mechanical ventilator support, or received a high dose of sedative or neuromuscular blocking drugs. ${ }^{5}$

Mainly the clinicians involved in ICU patient management are physicians, anesthetists, and nurses. Since there are different health personnel they are doing in different ways. So this is one of the challenges faced in managing ICU patients. Sedation and analgesia for ICU patients should have a protocol to decrease interpersonal differences in managing critical patients. ${ }^{4}$

Adult ICU patients are critically ill patients who need special care and strictly follow up that needs standard protocol in every aspect of management. One of the important management for adult ICU patent is sedation and analgesia. Since in most ICU patients anxiety and pain is common, they need both sedation and analgesics depending on their level of anxiety/ agitation and severity of pain. There are internationally accepted sedation and analgesia guidelines for adult ICU patients. Lack of resources in a low resource setting was among challenges in the field of critical care. Some of the medications were not available during ICU care. Thus, finding alternative guides through a systemic review of literature is the main aim of this study. Among several issues in ICU care, monitoring sedation, level of sedation, pharmacology of sedatives, pain assessment and pain management will be discussed systematically.

\section{Methodology}

The review was reported based on preferred reporting items for systemic and meta-analysis (PRISMA) protocol. We searched articles published in English from PubMed, Google Scholar, and Medline database to identify studies for our review using the keywords; the level of sedation, sedation score, pain assessment in ICU, and sedative drugs in ICU. From a lot of journals obtained from a search engine, filtering was made based on the intervention, outcome, data on population, and methodological quality. Besides, articles with a pediatric patient population and sedation out of ICU were excluded from the review. After extraction with a patient population and exclusion, five randomized clinical trials, four systemic reviews and metaanalysis, four observation cohort study and two practical guidelines were used for the review using the keywords (light AND deep sedation) midazolam AND diazepam, midazolam AND Propofol, and ketamine for ICU sedation (see Figure 1). Articles were independently assessed and determined their eligibility based on methodological quality on inclusion and exclusion criteria, and outcome variables by two evaluators. Any discrepancy was corrected by the third evaluator. The conclusion was made based on the level of evidence that was referred from the Oxford Center for evidence-based medicine (Table 1).

\section{Discussion \\ Monitoring Sedation}

To monitor the level of sedation uses of a structured tool for assessment of sedation and agitation is necessary. It helps to minimize the dose of sedative drugs and to avoid agitated behaviors. Even though all sedation assessment tools have their limitation a clinical guideline for pain, agitation, and delirium of adult ICU in 2013 shows that Richmond agitation sedation (RASS) and (ASA) are the most valid and reliable sedation assessment scales for measuring both depths of sedation and quality of sedation in adult ICU patients. ${ }^{6}$

In the study that compares the validity and reliability of RASS with the ASA scale, it shows that RASS as logical, easy to remember, and simple for administration for nurses and also RASS has high validity and reliability in surgical and medical ICU patients regardless of sedation status and the need of ventilator support. Besides, it describes that RASS has an advantage in decreasing the dose of sedative medication and the length of mechanical ventilation. ${ }^{7,8}$ This tool had also demonstrated excellent interrater reliability and criterion, construct, and face reliability and validity. ${ }^{9}$

Despite its inherent limitations, sedation monitoring tools like Frontalis electromyogram, Lower esophageal contractility, Continuous electroencephalography, Power spectral analysis, Sensory and auditory evoked potentials are not easily available in a low resource setting. In addition to scarce resources, the utility and practice of this different tool and techniques in the daily ICU setting is unknown. ${ }^{10}$

It is highly recommended that using RASS is an effective sedation assessment tool for clinical use in adult ICU.

\section{Level of Sedation}

The practice of light and deep sedation has an impact on cognitive and mental health function in ICU patients 

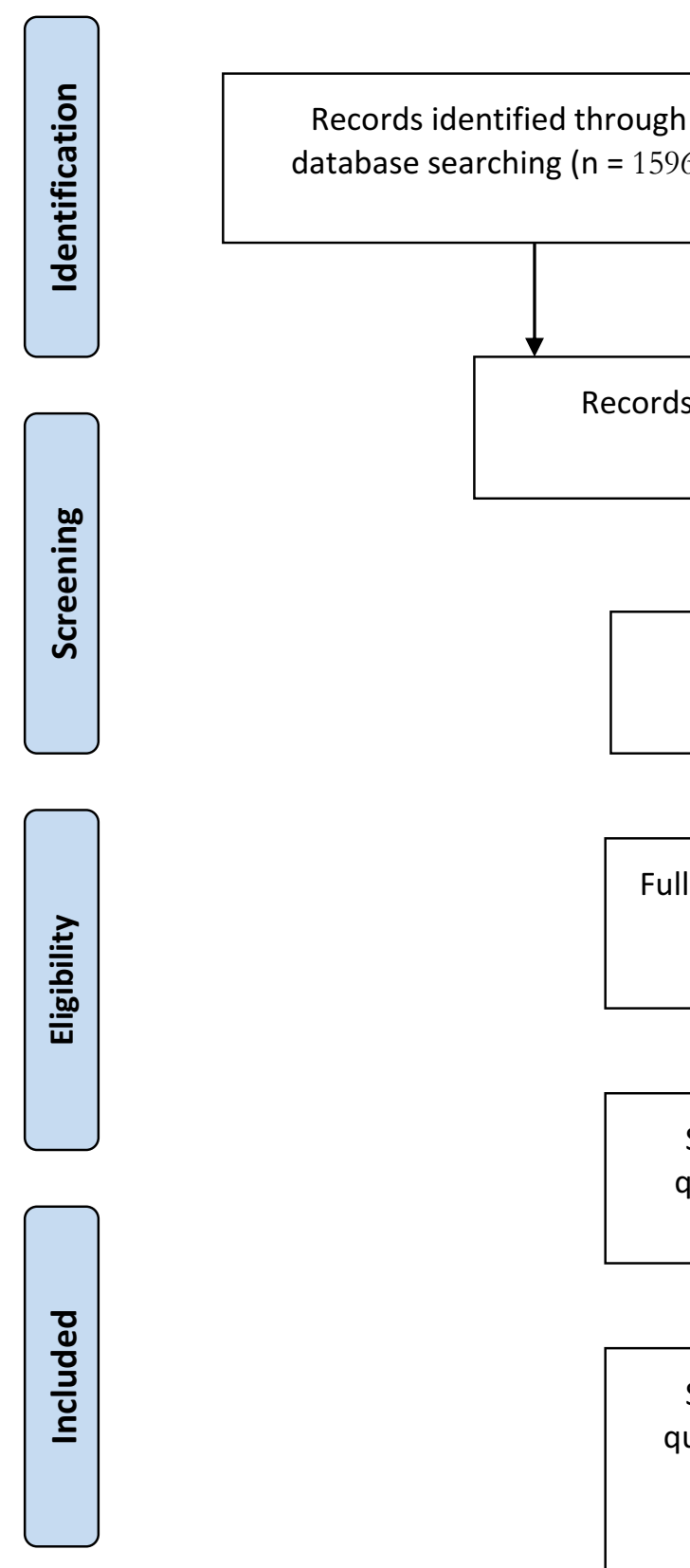

Additional records identified through other sources $(n=14)$ database searching $(n=1596)$

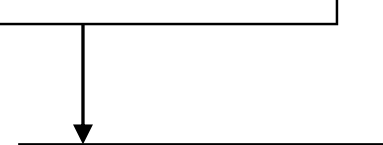

Records excluded $(n=4)$

Records after duplicates removed $(n=49)$
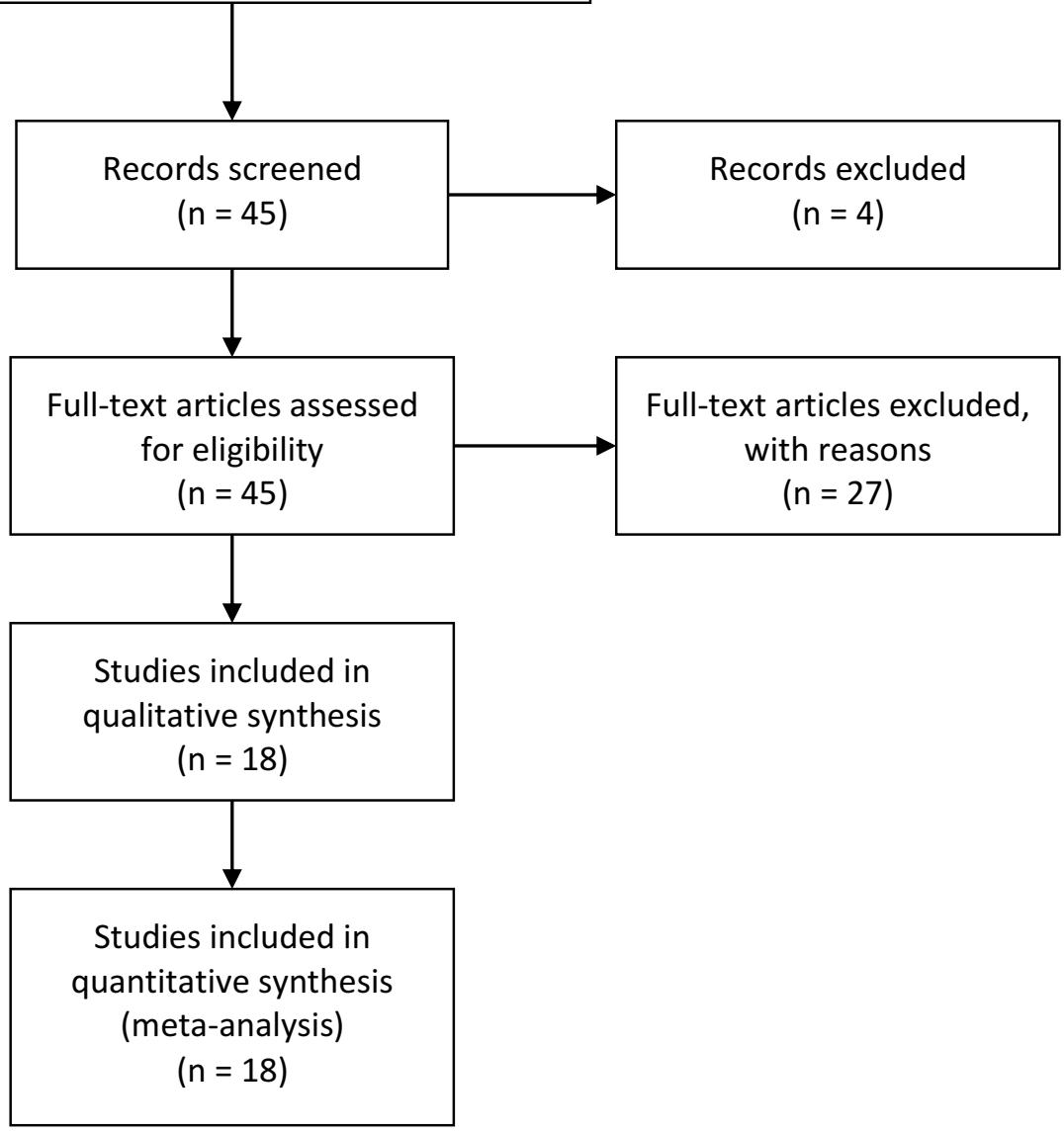

Figure I PRISMA Flow chart for selection of studies.

Notes: PRISMA figure adapted from Liberati A, Altman D, Tetzlaff J et al. The PRISMA statement for reporting systematic reviews and meta-analyses of studies that evaluate health care interventions: explanation and elaboration. Journal of Clinical Epidemiology. 2009;62(I0). Creative Commons.

both medically and psychologically. Sedation can be light in which the patient is in an arousal state and can purposefully respond for simple instructions or deep in which the patient is not responding to pain full stimuli. A meta-analysis revealed a significantly higher incidence of cognitive dysfunction in deep sedation compared to a light level of sedation and the conclusion was a deep level of sedation can result in cognitive impairment in adult ICU patients. ${ }^{8}$
Also, a randomized controlled trial was done in 2012 to support the light level of sedation and daily interruption of sedation to maintain the patient's responsiveness, improve communication, and it was also encouraged daily interruption of sedation. ${ }^{11}$

In the same way, another randomized control trial study which compared light versus deep sedation recommended light sedation because it minimizes ventilator support time by one (1 day) and ICU stay time by one 
Table I Levels of Evidences and Grades of Recommendations

\begin{tabular}{|l|l|l|}
\hline $\begin{array}{l}\text { Level of } \\
\text { Evidences }\end{array}$ & Grading Criteria & $\begin{array}{l}\text { Grade of } \\
\text { Recommendation }\end{array}$ \\
\hline Ia & $\begin{array}{l}\text { Systematic reviews of RCTs } \\
\text { including meta-analysis }\end{array}$ & A \\
\hline Ib & $\begin{array}{l}\text { Individual RCT with narrow } \\
\text { confidence interval }\end{array}$ & A \\
\hline Ic & $\begin{array}{l}\text { All or none randomized } \\
\text { controlled trials }\end{array}$ & B \\
\hline $2 \mathrm{~b}$ & $\begin{array}{l}\text { Systematic review of cohort } \\
\text { study } \\
\text { Individual cohort including low } \\
\text { quality RCT }\end{array}$ & B \\
\hline $2 \mathrm{c}$ & Outcome research study & C \\
\hline $3 \mathrm{a}$ & $\begin{array}{l}\text { Systematic review of case } \\
\text { control studies }\end{array}$ & C \\
\hline $3 \mathrm{~b}$ & $\begin{array}{l}\text { Individual case control study } \\
\text { Case series, poor quality } \\
\text { cohort and case control } \\
\text { studies }\end{array}$ & $\begin{array}{l}\text { Cxpert opinion without explicit } \\
\text { critical appraisal, or based on } \\
\text { physiology, bench research or } \\
\text { "first principles" }\end{array}$ \\
\hline 4 & C & \\
\hline 5 & RCT, Randomied Cicica Trial & \\
\hline
\end{tabular}

Abbreviation: RCT, Randomized Clinical Trial.

Note: Adapted from Centre for Evidence-Based Medicine (https://creativecom mons.org/licenses/by/4.0/). ${ }^{24}$

and halve $\left(1 \frac{1}{2}\right)$ day without negatively affecting patients mental and physical health. The study reveals no significant changes in the occurrences of anxiety, depression, mortality, and other adverse events. ${ }^{12}$

The 2013 Clinical practice guidelines for the management of pain, agitation in ICU patients also suggest light sedation for critically ill, mechanically ventilated patients. ${ }^{6}$ Excessive sedation even for less than 48 hours can increase morbidity and mortality as shown in a prospective cohort study done on critically ill patients. ${ }^{13}$

It was recommended that light sedation with daily interruption of sedative infusion or titration of a sedative dose with the final goal of having awake and alert patients who could perform a weaning trial unless there is a contraindication.

\section{Pharmacology of Sedatives}

An ideal sedative agent should have a fast onset, a short duration of action, a lack of accumulation, ease of titration, and administration, without cardiovascular and respiratory depression. ${ }^{14}$ But the absence of this ideal sedative result in a different method of sedation in ICU patients was the basis for variations among published guidelines and practical variations. Among agents commonly used for ICU sedation diazepam, midazolam, Propofol, and ketamine as an induction agent were revised.

Benzodiazepines such as midazolam and lorazepam are among commonly used sedatives in ICU. Propofol has been another alternative commonly used to sedate adult critically ill patients in most cases. Midazolam was the most popularly utilized drug for sedation (65-70\% of the time), Propofol used $20 \%$ of the time. According to, open-label trial, compared to midazolam and fentanyl, Propofol and remifentanil have been shown to facilitate a higher turnover of patients by reducing the time of mechanical ventilation and also shortening ICU stay time. The trial revealed the higher cost of remifentanil had been compensated by shorter ICU stay. ${ }^{15}$

The 2002 guidelines recommend midazolam should only be used for short-term sedation, while lorazepam should be considered only for long-term sedation. Propofol, however, shall be used for patients requiring intermittent awakenings. ${ }^{16}$

However, when long-term sedation is required, the toxic accumulation associated with an agent like Propofol helps in finding alternative sedative agents. Ketamine, a non-barbiturate, phencyclidine derivative commonly accessed in a low resource setting could be a viable alternative for continuous sedation. ${ }^{17}$

\section{Propofol vs Midazolam}

A systemic review indicates that propofol is similarly effective as midazolam but it provides predictable and faster recovery even after prolonged infusion $(>72$ hours). It was also reported that control of the depth of sedation is easily achieved by propofol sedation. The review finally concludes that propofol sedation effective sedation with a more rapid and predictable emergence time than midazolam in a variety of clinical settings. ${ }^{18}$

In the same way, another study reported that Propofol and midazolam are similarly effective in providing the required level of sedation. However, after the decision of weaning made, Propofol minimizes the time to extubation regardless of the duration of sedation. ${ }^{14}$

A meta-analysis shows no significant difference in mortality between sedation with Propofol and other different sedative agents with odds ratio [OR] $1.05,95 \%$ confidence interval [CI] $0.80-1.38, \mathrm{P}=0.74$; I $(2)=(0 \%)$. It shows Using medium and long-term sedation with 


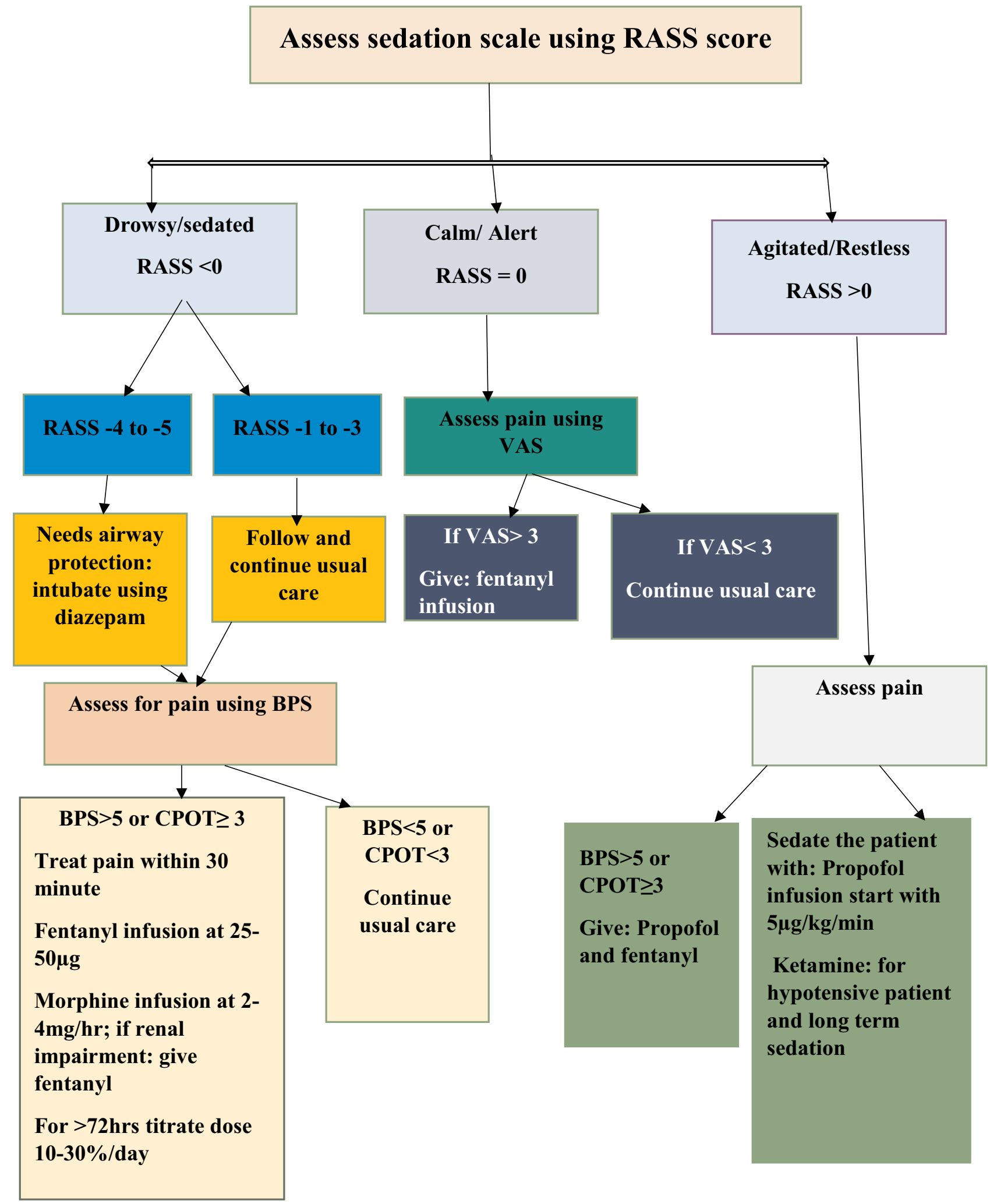

Figure 2 Flow chart for adult sedation, pain assessment, and analgesia in an intensive care unit.

Abbreviations: BPS, Behavioral Pain Scale; CPOT, Critically-ill Pain Observation Tool; RASS, Richmond Agitation and Sedation Scale; VAS, Visual Analogue Scale. 
propofol was associated with a significant reduction in length of ICU stay with overall weighted-meandifference [WMD] in days $-0.99,95 \% \mathrm{CI}-1.51$ to $-0.47, \mathrm{P}=0.0002 ; \mathrm{I}(2)=(82.26 \%)$ when compared to different sedative agents but it is insignificant when compared with midazolam. ${ }^{19}$

Propofol is recommended for short-term sedation and preferable to midazolam and other sedative drugs. Also for medium- and long-term sedation, it is as safe as midazolam even for more than 72 hrs. So we can use Propofol both for short-term and long-term sedation safely.

\section{Ketamine for ICU Sedation}

Systemic review shows that ketamine can be used as an alternative for sedation in adult ICU patients and it has an advantage for hypotensive patients and also for patients requiring a high dose of vasopressors. The study shows that it is an effective sedative agent as fentanyl was for head injury patient. Ketamine can be used as an alternative sedative agent in case of long-term sedation where midazolam and Propofol may result in toxic accumulation or Propofol infusion syndrome. ${ }^{17}$

Ketamine is also recognized by beneficial respiratory and cardiovascular effects when administered as maintenance sedation via continuous infusion. The effect of ketamine is on Functional residual capacity, minute ventilation, tidal volume is very minimal compared to other sedatives. Furthermore, it was shown to decrease airway resistance and preserve protective pharyngeal and laryngeal reflexes in an asthmatic patient with refractory bronchospasm. Administration of ketamine by continuous infusion has been reported to decrease audible wheezing, carbon dioxide level, bronco dilator requirements, and improve respiratory rate and oxygenation. ${ }^{20}$ It is also recommended to use ketamine as an alternative when continuous sedation for a long time is required to decrease the side effect of others. ${ }^{17}$

It is recommended to use ketamine as an alternative sedative agent in adult ICU especially for patients with asthma and hypotensive patients. It is also recommended for longer continuous sedation.

\section{Regarding to Diazepam or Midazolam}

A randomized controlled trial study that compared clinically and cost-effectiveness between diazepam and midazolam for urgent intubation in ICU indicates that time for adequate sedation was shorter with $132 \pm 87$ sec for diazepam vs. $224 \pm 117 \mathrm{sec}, \mathrm{p}=0.016$ for midazolam. But the duration of sedation was similar to $86 \pm 67 \mathrm{~min}$ vs. $88 \pm 50$ min, $\mathrm{p}=0.936$ for diazepam when compared to midazolam.
The total dose of drugs to reach adequate sedation is similar for both drugs (10-12 mg vs. 10-17 mg, $\mathrm{p}=0.248)$. Cost of sedation was lower for diazepam compared to midazolam $(1.4-1.8$ vs. $9.4-16.2, \mathrm{p}<0.001) .{ }^{21}$ It is recommended to use diazepam in adult ICU for the patient who needs urgent intubation.

\section{Pain Assessment in ICU}

Pain is a protective mechanism for a certain pathophysiologic condition that needs to be corrected but also a cause for unwanted stressor of those pathophysiologic problems. Pain in addition to negative effects on normal physiology can cause anxiety in critically ill patients. Measurement of pain in ICU especially unconscious patients can be challenging. So several tools for measuring the pain for critically ill patients already developed and validated. A prospective observational study shows that for non-communicating patients the common pain assessment tool used is BPS (Behaviour Pain Scale) and CPOT (Critically-ill Pain Observation Tool). BPS has three parameters that are facial expression, upper limb movement, and compliance with ventilator, while CPOT uses four parameters that are facial expression, muscular tone (passive movement), upper extremity movement (active), and compliance with the ventilator. It was indicated in the study that CPOT and BPS were a good criterion and discriminant validity $(p<0.0001)$. BPS was found to be more specific (91.7\%) than CPOT (70.8\%), but less sensitive (BPS 62.7\%, CPOT 76.5\%). COPT and BPS scores have a significant correlation with VAS $(p<0.0001)$. The combination of BPS and CPOT provides better sensitivity of $80.4 \%$. Facial expression was the main parameter to determine pain scale changes effect size $=1.4 .^{22}$ For conscious patients who can self-report VAS is the gold standard for evaluation of pain their pain and $\mathrm{VAS} \geq 3$ is used to determine patient with pain. ${ }^{23}$

It is recommended to use a combination of BPS (Behaviour Pain Scale) and CPOT (Critically-ill Pain Observation Tool) for critically ill mechanically ventilated adult ICU patients. And for conscious patients use VAS.

\section{Pain Management in Adult ICU}

A clinical practice guideline for the management of pain in adult ICU which is revised in 2013 recommends that preemptive analgesia should be administered to alleviate pain in adult ICU before chest tube removal and it recommends IV opioids to be considered as the first line of choice to treat non-neuropathic pain in critically ill patients. And all available opioids when titrated to similar pain intensity 
endpoints are equally effective. Also, it recommends that non-opioid analgesics should be considered to decrease the dose of opioids administered and to decrease the side effect of opioids. ${ }^{6}$ We can adopt pain management protocols from the guideline of management of pain in adult ICU which was revised in 2013.

\section{Conclusion}

In addition to high validity and reliability, RASS has the advantage of easiness to remember for nurses making it a preferred sedation assessment tool in an adult ICU setting. The level of sedation when in deep was highly associated with mortality and morbidity. Thus, light sedation with daily interruption was recommended with an aim of an awake and alert patient ready for the weaning trial. Propofol was preferred when sedation is for a short duration and when intermittent awakening is required. For patients with a co-existing disease such as asthma, ketamine was alternatives. Ketamine is also recommended for hypotensive patient and a long-term continuous sedation. With a similar time for sedation, diazepam shows a shorter time for intubation compared to midazolam. Besides diazepam has shown a cheaper cost of sedation than midazolam. This makes it a drug of preference in a low resource setting. A combination of BPS (Behaviour Pain Scale) and CPOT (Critically-ill Pain Observation Tool) was recommended for pain assessment of critically ill mechanically ventilated adult ICU patients. And for conscious patients use VAS (see Figure 2).

\section{Registration}

It was sent for registration.

\section{Abbreviations}

ASA, American Society of Anesthesiologists; BPS, Behavioral Pain Scale; CI, confidence interval; CPOT, Critically-ill Pain Observation Tool; ICU, intensive care unit; RASS, Richmond Agitation and Sedation Scale; PRISMA, Preferred Reporting Items for Systemic Review and MetaAnalysis; PSA, procedural sedation and analgesia; VAS, Visual Analogue Scale; WMD, weighted mean difference.

\section{Ethical Approval}

Ethical approval is not required.

\section{Consent for Publication}

Not applicable.

\section{Funding}

No funding was obtained from any organization.

\section{Disclosure}

The authors declare that they have no competing interests.

\section{References}

1. Chanques G, Jaber S, Barbotte E, et al. Impact of systematic evaluation of pain and agitation in an intensive care unit. Crit Care Med. 2006;34(6):1691-1699. doi:10.1097/01.CCM.0000218416.62457.56

2. Chanques G, Sebbane M, Barbotte E, Viel E, Eledjam JJ, Jaber S. A prospective study of pain at rest: incidence and characteristics of an unrecognized symptom in surgical and trauma versus medical intensive care unit patients. J Am Soc Anesthesiolog. 2007;107(5):858-860.

3. Seitz DP, Gill SS, Zyl LTV. Antipsychotics in the treatment of delirium: a systematic review. J Clin Psychiatr. 2007;68(1):11-21. doi:10.4088/JCP.v68n0102

4. Schweickert WD, Pohlman MC, Pohlman AS, et al. Early physical and occupational therapy in mechanically ventilated, critically ill patients: a randomized controlled trial. Lancet (London, England). 2009;373(9678):1874-1882. doi:10.1016/S0140-6736(09)60658-9

5. Ahlers SJ, van Gulik L, van der Veen AM, et al. Comparison of different pain scoring systems in critically ill patients in a general ICU. Critical Care. 2008;12(1):R15. doi:10.1186/cc6789

6. Barr J, Fraser GL, Puntillo K, et al. Clinical practice guidelines for the management of pain, agitation, and delirium in adult patients in the intensive care unit. Crit Care Med. 2013;41(1):263-306. doi:10.1097/CCM.0b013e3182783b72

7. Yousefi H, Toghyani F, Yazdannik A, et al. Effect of using the Richmond Agitation Sedation Scale on the duration of mechanical ventilation, type, and dosage of sedation on hospitalized patients in intensive care units. Iran J Nurs Midwifery Res. 2015;20(6):700. doi:10.4103/1735-9066.170008

8. Porhomayon J, A Joude P, Adlparvar G, et al. The impact of high versus low sedation dosing strategy on cognitive dysfunction in survivors of intensive care units: a systematic review and meta-analysis. J Cardiovasc Thorac Res. 2015;7(2):43. doi:10.15171/jcvtr.2015.10

9. Ely EW, Truman B, Shintani A, et al. Monitoring sedation status over time in ICU patients: reliability and validity of the Richmond Agitation-Sedation Scale (RASS). JAMA. 2003;289(22):2983-2991. doi:10.1001/jama.289.22.2983

10. Carrasco G. Instruments for monitoring intensive care unit sedation. Critical Care. 2000;4(4):217. doi:10.1186/cc697

11. Mehta S, Burry L, Cook D, et al. Daily sedation interruption in mechanically ventilated critically ill patients cared for with a sedation protocol: a randomized controlled trial. JAMA. 2012;308 (19):1985-1992. doi:10.1001/jama.2012.13872

12. Treggiari M. Randomized trial of light versus deep sedation on mental health after critical illness. Crit Care Med. 2010;38 (1):349-350. doi:10.1097/CCM.0b013e3181c546f8

13. Shehabi Y, Chan L, Kadiman S, et al. Sedation depth and long-term mortality in mechanically ventilated critically ill adults: a prospective longitudinal multicentre cohort study. Intensive Care Med. 2013;39 (5):910-918. doi:10.1007/s00134-013-2830-2

14. Jacobi J, Fraser GL, Coursin DB, et al. Clinical practice guidelines for the sustained use of sedatives and analgesics in the critically ill adult. Crit Care Med. 2002;30(1):119-141. doi:10.1097/00003246200201000-00020

15. Muellejans B, Matthey T, Scholpp J, Schill M. Sedation in the intensive care unit with remifentanil/propofol versus midazolam/fentanyl: a randomized, open-label, pharmacoeconomic trial. Critical Care. 2006;10(3):R91. doi:10.1186/cc4939 
16. Fraser GL, Devlin JW, Worby CP, et al. Benzodiazepine versus nonbenzodiazepine-based sedation for mechanically ventilated, critically ill adults: a systematic review and meta-analysis of randomized trials. Crit Care Med. 2013;41(9):S30-S38. doi:10.1097/ CCM.0b013e3182a16898

17. Umunna BP, Tekwani K, Barounis D, Kettaneh N, Kulstad E. Ketamine for continuous sedation of mechanically ventilated patients. J Emerg Trauma Shock. 2015;8(1):11. doi:10.4103/09742700.145414

18. Zhou Y, Jin X, Kang Y, et al. Midazolam and propofol used alone or sequentially for long-term sedation in critically ill, mechanically ventilated patients: a prospective, randomized study. Critical Care. 2014;18(3):R122. doi:10.1186/cc13922

19. Ho KM, Ng JY. The use of propofol for medium and long-term sedation in critically ill adult patients: a meta-analysis. Intensive Care Med. 2008;34(11):1969. doi:10.1007/s00134-008-1186-5

20. Miller A, Jamin C, Elamin E. Continuous intravenous infusion of ketamine for maintenance sedation. Minerva Anestesiol. 2011;77 (8):812-820.
21. Gehrke L, Oliveira RP, Becker M, et al. Diazepam or midazolam for orotracheal intubation in the ICU? Revista Da Associação Médica Brasileira. 2015;61(1):30-34. doi:10.1590/1806-9282.61.01.030

22. Chanques G, Viel E, Constantin J-M, et al. The measurement of pain in the intensive care unit: comparison of 5 self-report intensity scales. PAIN ${ }^{\circledR}$. 2010;151(3):711-721. doi:10.1016/j.pain.2010.08.039

23. Severgnini P, Pelosi P, Contino E, et al. Accuracy of Critical Care Pain Observation Tool and Behavioral Pain Scale to assess pain in critically ill conscious and unconscious patients: prospective, observational study. J Intensive Care. 2016;4(1):68. doi:10.1186/s40560016-0192-x

24. Centre for Evidence-Based Medicine. Oxford Centre for EvidenceBased Medicine: Levels of Evidence (March 2009). Oxford: University of Oxford; 2009. Available from: https://www.cebm.ox. ac.uk/resources/levels-of-evidence/oxford-centre-for-evidence-basedmedicine-levels-of-evidence-march-2009. Accessed December 3, 2020.

\section{Publish your work in this journal}

The International Journal of General Medicine is an international, peer-reviewed open-access journal that focuses on general and internal medicine, pathogenesis, epidemiology, diagnosis, monitoring and treatment protocols. The journal is characterized by the rapid reporting of reviews, original research and clinical studies across all disease areas. The manuscript management system is completely online and includes a very quick and fair peer-review system, which is all easy to use. Visit http://www.dovepress.com/ testimonials.php to read real quotes from published authors. 